\title{
Southern Analysis after Long-range PCR: Clinical Appli- cation in Korean Patients with Myotonic Dystrophy 1
}

Mi-Sun Yum, Beom Hee Lee ${ }^{1,2}$, Gu-Hwan Kim², Jin-Joo Lee ${ }^{2}$, Seung Hoon Choi ${ }^{2}$, Joo Yeon Lee², Jae-Min Kim², Yoo-Mi Kim¹, TaeSung $\mathrm{Ko}^{1}$ and Han-Wook Yoo ${ }^{1,2 *}$

${ }^{1}$ Department of Pediatrics, ${ }^{2}$ Medical Genetics Center, Asan Medical Center Children's Hospital, University of Ulsan College of Medicine, Seoul, Korea

Purpose: Myotonic dystrophy 1 (DM1, OMIM 160900) is an autosomal-dominant muscular disorder caused by an expansion of CTG repeats in the 3' UTR of the DMPK gene. Variable expansions of CTG repeats preclude the accurate determination of repeat size. We tried to show the clinical and analytical validity of the application of Southern blotting after long-range PCR was demonstrated in Korean DM1 patients.

Materials and Methods: The Southern blotting of long-range PCR was applied to 1,231 cases with clinical suspicion of DM1, between 2000 and 2011. PCR was performed using genomic DNA with forward 5'-CAGTTCACAACCGCTCCGAGC-3' and reverse 5'-CGTGGAGGATGGAACACGGAC-3' primers. Subsequently, the PCR fragments were subjected to gel electrophoresis, capillary transfer to a nylon membrane, hybridization with a labeled (CAG)10 probe. The correlation between clinical manifestations and the CTG repeat expansions were analyzed.

Results: Among a total of 1,231 tested cases, 642 individuals were diagnosed with DM1 and the range of the detected expansion was 50 to 2,500 repeats; fourteen cases with mild DM1 (75 \pm 14 repeats), 602 cases with classical DM1 (314 \pm 143 repeats), and 26 cases with congenital DM1 (1,219 \pm 402 repeats). The positive and negative predictive values were $100 \%$. The age at test requested and the CTG repeat numbers were inversely correlated ( $R=-0.444, P<0.01)$.

Conclusion: This study indicates that Southern blotting after long-range PCR is a reliable diagnostic method DM1.

Key words: Myotonic dystrophy 1, Southern blotting, Long-range PCR, Genetic testing

\section{Introduction}

Myotonic dystrophy 1 (DM1, OMIM 160900) is one of the most common muscular dystrophies, inherited in an autosomal-dominant manner, and is typically characterized by myotonic myopathy but also involves multiple organs, including eye, heart, endocrine system, and the central nervous system. The clinical phenotype is highly variable and classified into three subtypes according to severity: mild; classical; and congenital.' The abnormal expansion of a trinucleotide CTG repeat in the 3'UTR of the DMPK1 gene is the underlying molecular cause of $\mathrm{DM} 1^{2)}$ and diagnosis is based on detection of these abnormally expanded repeats. There is a clear association between CTG repeat size and age at onset and clinical severity ${ }_{i}^{1,3-5)}$ it is important, therefore, to identify the accurate

\footnotetext{
Received: 28 May 2013, Revised: 9 June 2013, Accepted: 12 June 2013, Published: 30 June 2013

*Corresponding author: Han-Wook Yoo, M.D., Ph.D.

Department of Pediatrics and Medical Genetics Asan Medical Center, University of Ulsan College of Medicine 388-1, Poongnap-dong, Songpa-ku, Seoul, 138-736, Korea

Tel: 82-2-3010-3390, Fax: 82-2-473-3725, E-mail: hwyoo@amc.seoul.kr

${ }^{*}$ Conflict of interest: We declare that we do not have any conflicts of interests.

(c) This is an open-access article distributed under the terms of the Creative Commons Attribution Non-Commercial License (http://creativecommons.org/licenses/by-nc/3.0/) which permits unrestricted non-commercial use, distribution, and reproduction in any medium, provided the original work is properly cited.

(c) Copyright 2013 by the Korean Society of Medical Genetics www.e-kjgm.org
} 
number of CTG repeats to facilitate appropriate prediction of clinical severity and outcome. The range of CTG repeat numbers is 50 to 150,100 to 1,000 , and 1,000 to > 2,000 in mild, classic, and congenital DM1, respectively, and this wide variation in expansion of CTG repeats has led to difficulty in establishing a clinically reliable test. ${ }^{6,}$

${ }^{7)}$ Additionally, variable penetrance and somatic mosaicism among patients preclude the determination of accurate repeat size. In this study, we used Southern analysis after long-range PCR of genomic DNA for the diagnosis of DM1.

\section{Materials and Methods}

Southern analysis after long-range PCR was applied to cases referred for genetic testing of DM1 at the Asan Medical Center between 2000 and 2011. Genomic DNA from peripheral blood was used as a template for PCR amplification. Long-range PCR was performed using LA-Taq polymerase (TAKARA, Shiga, Japan) with forward (5'-CAGTTCACAACCGCTCCGAGC-3') and reverse (5'-CGTGGAGGATGGAACACGGAC-3') primer set. The long-range PCR products were resolved on 1\% agarose gel electrophoresis $(75 \mathrm{~V}, 2 \mathrm{hr})$, and transferred to a nylon membrane and fixed at $80^{\circ} \mathrm{C}$ for $1 \mathrm{~h}$. Hybridization was performed at $45^{\circ} \mathrm{C}$ using biotinlabeled (CTG) 10 probe overnight. After washing and incubation in blocking solution for $50 \mathrm{~min}$, membranes were stained with $2 \mu \mathrm{L}$ alkaline phosphatase labeled streptoavidine for $50 \mathrm{~min}$. CDP-Star chemiluminescent substrate ( $K P L, M D, U S A)$ treated blots were exposed to X-ray film or detected by ChemiDoc XRS+ (BioRad, CA, USA). The expanded alleles revealed a smeared band and the size of the allele with the strongest density were reported as shown in Fig. ${ }^{8)}$

To ensure the accuracy of this Southern blot of long-range PCR test for DM1, we confirmed the test protocol using the 54 controls (18 positive controls with range; $400-650$ repeats, 36 negative) of the Collage of American Pathology (CAP). The positive rates of the diagnosis were determined. The size of CTG repeats was presented as mean \pm standard deviation (SD). To delineate the possible correlation between genotype and phenotype, the relationship between the age at test requested and the size of CTG repeats was also analyzed using Pearson's correlation analysis. SPSS 18.0 was used for the statistical analysis.

\section{Results}

To ensure the accuracy of this method, we validated our test using 54 controls (18 positive) obtained from the CAP and all controlswereaccurately determined. The number of CTG repeats was underestimated by $140 \pm 88$ in our test compared to CAP data. Among 1,231 referred cases, 642 patients (52.5\%) were diagnosed with DM1. The age at diagnosis of DM1 and the number of CTG repeats were inversely correlated ( $R=-0.444, P<0.01)$ (Fig. 2). Fourteen cases were diagnosed with mild $\mathrm{DM} 1$, and their repeat size was $76 \pm 14$, the CTG repeat size of 602 cases with classical DM1 was $314 \pm 143$, and that of 26 cases with congenital DM1 was $1,219 \pm 402$ (Table 1). Maternal anticipation was also demonstrated in all 17 familial cases of DM1. The offspring showed CTG repeat expansions of a median of 800 (range, 50-1,950) compared to their mother.

\section{Discussion}

DM1 is the most common muscular dystrophy in adults with an estimated incidence of 1 in 8,000 individuals. ${ }^{2,9)}$ The DMPK gene, which is containing 14 or 15 exons and encoding a serinethreonine kinase expressed in all tissues, is the only known gene associated with DM1. The diagnostic molecular test for DM1 is aimed to detect the unstable hyperextensions of the CTG triplet repeat in the DMPK gene.

In patients with clinical suspicion of muscular dystrophies, the confirmation of etiological diagnosis is very important, not only

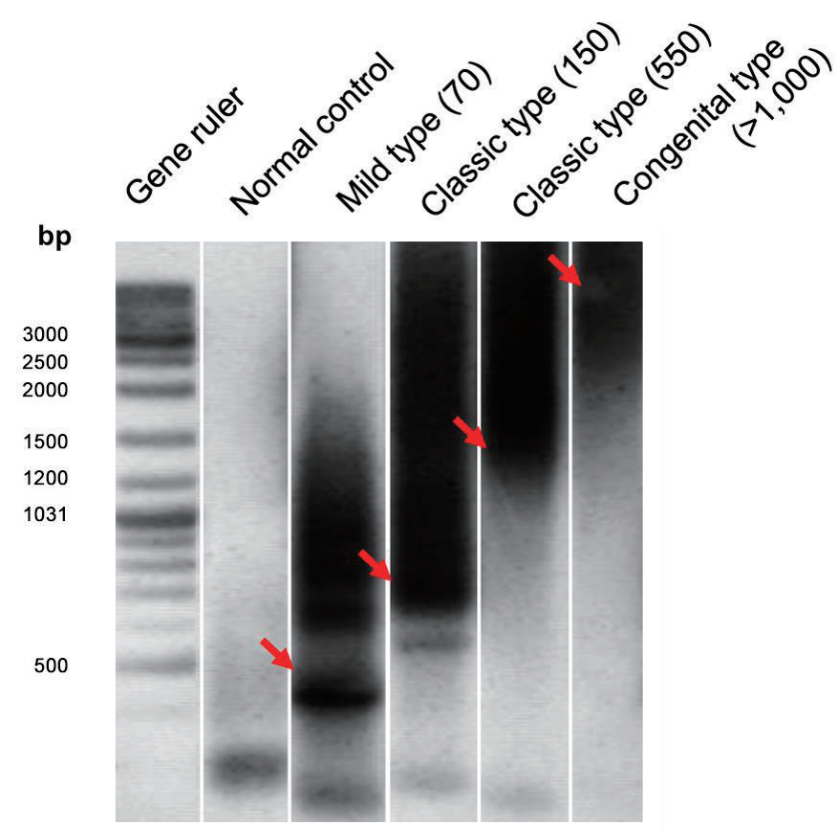

Fig. 1. Southern blot of long-range PCR products from the CTG repeat in the DMPK gene. Lanes contain samples from a normal control and patients with mild, classical, and congenital types of myotonic dystrophy. 


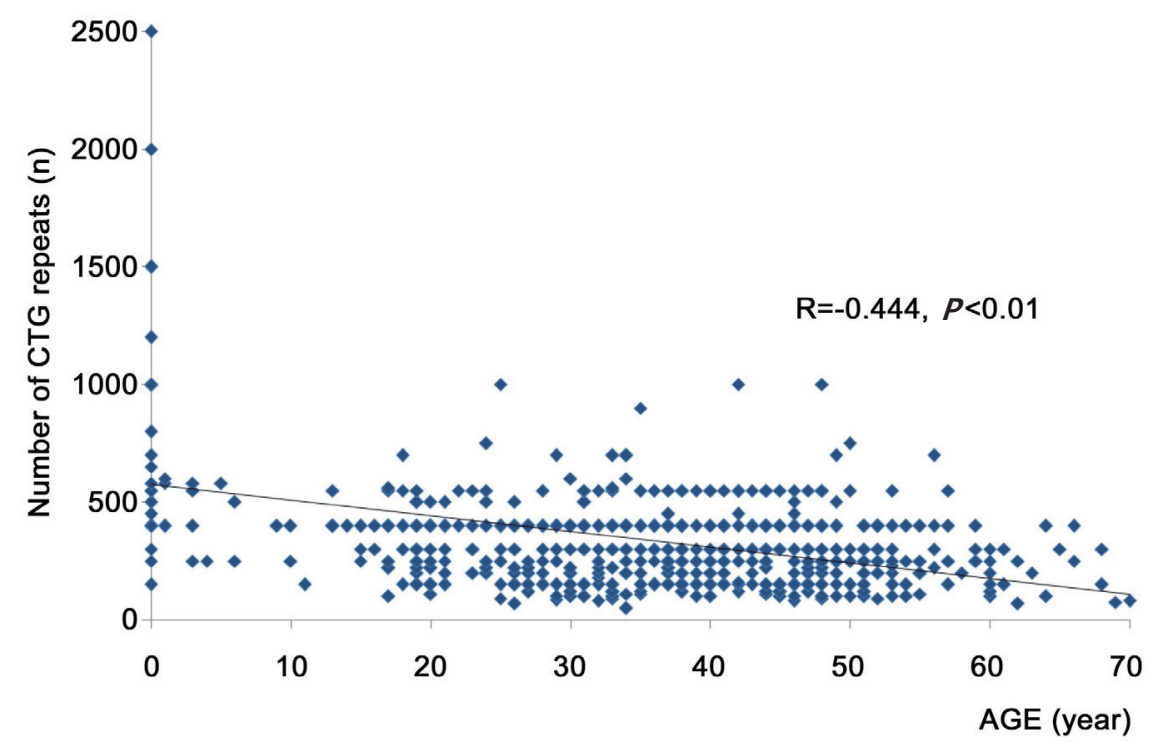

Fig. 2. The relationship between age at diagnosis and (CTG) $)_{n}$ repeat expansion in the DMPK gene.

Table 1. The Distribution of (CTG) $)_{n}$ Expansions According DM1 Subtype

\begin{tabular}{lcc}
\hline Phenotype (range of $\left.(\mathrm{CTG})_{n}{ }^{*}\right)$ & Number of subjects & $(\mathrm{CTG})_{n}$ by Southern blotting after Long-range PCR \\
\hline Normal $(5$ to 35$)$ & 589 & - \\
Mild $(-50$ to -150$)$ & 14 & $75 \pm 14$ (range, 50 to 90 repeats) \\
Classic $(-100$ to $-1,000)$ & 602 & $313 \pm 143$ (range, 100 to 900 repeats) \\
Congenital $(-1,000$ to 2,000$)$ & 26 & $1,219 \pm 402$ (range, 1,000 to $-2,500$ repeats) \\
\hline
\end{tabular}

${ }^{\star}$ GeneReviews: http://www.ncbi.nlm.nih.gov/books/NBK1165/ ${ }^{13)}$

for the appropriate therapies but also for the counseling about the prognosis and family planning. Especially for the prenatal genetic diagnosis, the reliability and speed is critical. ${ }^{10)}$ Currently, several molecular diagnostic methods are available for the genetic diagnosis of DM1; however, the accurate determination of expansion size using a single method is frequently problematic. Therefore, combined testing, including both PCR and Southern blot, has been recommended for routine genetic testing. ${ }^{1,8} \mathrm{PCR}$ is a rapid test for the discovery of small expansions ( $<100$ repeats); however, longer repeat arrays $(>1,000)$ are not readily detected by PCR, hence Southern blotting using genomic DNA should be performed to detect these. Southern blotting using genomic DNA is a labor-intensive, time-consuming, and expensive process requiring high-quality DNA for adequate analysis. ${ }^{6,7)}$

The results of our study indicate that the Southern analysis after long-range PCR is a very useful method for the diagnosis of DM1, with high specificity and positive predictive value. This method is faster and less expensive than routine PCR \& fragment analysis and/or genomic Southern blots. The maximum detection limit was 2,500 CTG repeats, which is comparable with previous reports using small sample sizes. ${ }^{11,12)}$
The number of CTG-repeat units in stable nonpathogenic $D M P K$ alleles ranges from 5 to $34^{1,8)}$ and there is significant genotypephenotype correlation between the CTG repeat size and clinical severity. Infants with congenital DM1, floppy infants suffering respiratory insufficiency, failure to thrive, mental retardation and developmental delay, usually has more than 1,000 CTG repeats expansions. On the other hand, the mild forms of DM1, only comprising premature cataracts and baldness, have 50-150 CTG repeats. Using this correlation, the clinical phenotypes classified according to the clinical presentations can be determined by the CTG repeat size, which permits the prediction of clinical course. ${ }^{8}$ ${ }^{9}$, 13) Thus, the purpose of the molecular testing is not only proving the presence or absence of the expanded alleles but also the correct sizing of the CTG repeats. However, there is a repeat size overlap in patients with different clinical classifications and dynamic somatic mosaicism of CTG repeat toward continuous expansion throughout the life time of an affected individual.

Although Southern analysis after long-range PCR is a wellestablished technique, appropriate conditions to guarantee the amplification of GC-rich templates are required. Because long and GC-rich templates tend to form extremely stable 
secondary structures, which interfere with conventional PCR amplification, ${ }_{1}^{14)}$ it is very difficult to determine the optimal PCR conditions for diagnosis. In this study, we were able to establish the appropriate conditions for Southern analysis after longrange PCR for DM1 patients. To date, triplet repeat primed PCR (TP-PCR) has proven to be accurate and is used in many centers as an alternative to Southern analysis after long-range PCR. ${ }^{1,15)}$ Although expansions in all size ranges can be detected by TP-PCR, the determination of the expanded repeat length is unreliable because of the attenuation of the signal for longer repeats. ${ }^{16,}$ 17) Similar to TP-PCR, the numbers of CTG repeats observed by Southern analysis after long-range PCR have previously been reported to be too obscure. ${ }^{6,12,18)}$ Despite this difficulty of correct determination of largely expanded alleles, these issues did not affect the diagnosis and subtype classification of the patients. Additionally, we could also confirm that the age at diagnostic test were inversely correlated with the estimated numbers of CTG repeats by Southern blot of long-range PCR. The genetic anticipa$\operatorname{tion}^{19)}$ was also identified in 17 families with 4 times of repeat expansions, which also confirms the accuracy of the test.

The accuracy of Southern analysis after long-range PCR is low when testing for shortCTG repeat expansions and the differentiation of mild type DM1 from premutations was particularly difficult using this method. Although the differentiation of premutation and mild type DM1 was not critical for the clinical diagnosis in the symptomatic patient group, PCR \& fragment analysis should be done additionally for the diagnosis of carrier and asymptomatic family members of DM1.

This study has described precise PCR-Southern blot conditions that were successfully applied to clinical testing. This Southern analysis after long-range PCR is potentially applicable to other genetic disorders with repetitive GC-rich sequences.

\section{Acknowledgement}

We thank the patients and their families for participating in this study, which was supported by a grant from the Korean Ministry for Health, Welfare and Family Affairs (Grant No. A120367).

\section{References}

1. Kamsteeg EJ, Kress W, Catalli C, Hertz JM, Witsch-Baumgartner M, Buckley MF, et al. Best practice guidelines and recommendations on the molecular diagnosis of myotonic dystrophy types 1 and 2. Eur J Hum
Genet 2012;20:1203-8.

2. Turner $\mathrm{C}$, Hilton-Jones $\mathrm{D}$. The myotonic dystrophies: diagnosis and management. J Neurol Neurosurg Psychiatry 2010;81:358-67.

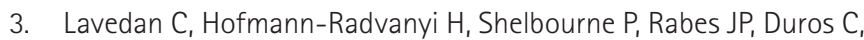
Savoy $D$, et al. Myotonic dystrophy: size- and sex-dependent dynamics of CTG meiotic instability, and somatic mosaicism. Am J Hum Genet 1993;52:875-83.

4. Harley HG, Rundle SA, MacMillan JC, Myring J, Brook JD, Crow S, et al. Size of the unstable CTG repeat sequence in relation to phenotype and parental transmission in myotonic dystrophy. Am J Hum Genet 1993; 52:1164-74.

5. Gennarelli M, Novelli G, Andreasi Bassi F, Martorell L, Cornet M, Mene-

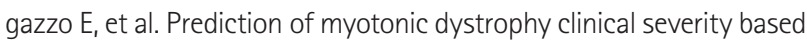
on the number of intragenic [CTG]n trinucleotide repeats. Am J Med Genet 1996;65:342-7.

6. Radvansky J, Kadasi L. The expanding world of myotonic dystrophies: how can they be detected? Genet Test Mol Biomarkers 2010;14:733-41.

7. Falk M, Vojtísková M, Lukás Z, Kroupová I, Froster U. Simple procedure for automatic detection of unstable alleles in the myotonic dystrophy and Huntington's disease loci. Genet Test 2006;10:85-97.

8. New nomenclature and DNA testing guidelines for myotonic dystrophy type 1 (DM1). The International Myotonic Dystrophy Consortium (IDMC). Neurology 2000;54:1218-21.

9. Harper PS. Myotonic dystrophy. 3rd ed. London: W.B.Sounders, 2001.

10. Zühlke C, Atici J, Martorell L, Gembruch U, Kohl M, Göpel W, et al. Rapid detection of expansions by PCR and non-radioactive hybridization: application for prenatal diagnosis of myotonic dystrophy. Prenat Diagn 2000;20:66-9.

11. Skrzypczak-Zielinska M, Sulek-Piatkowska A, Mierzejewski M, Froster UG. New analysis method of myotonic dystrophy 1 based on quantitative fluorescent polymerase chain reaction. Genet Test Mol Biomarkers 2009:13:651-5.

12. Cheng S, Barceló JM, Korneluk RG. Characterization of large CTG repeat expansions in myotonic dystrophy alleles using PCR. Hum Mutat 1996; 7:304-10

13. Bird TD. Myotonic Dystrophy Type 1. http://www.ncbi.nlm.nih.gov/ books/NBK1165/

14. Pearson CE, Sinden RR. Alternative structures in duplex DNA formed within the trinucleotide repeats of the myotonic dystrophy and fragile X loci. Biochemistry 1996;35:5041-53.

15. Radvansky J, Ficek A, Kadasi L. Upgrading molecular diagnostics of myotonic dystrophies: multiplexing for simultaneous characterization of the DMPK and ZNF9 repeat motifs. Mol Cell Probes 2011;25:182-5.

16. Hamzi K, Bellayou H, Slassi I, Nadifi S. A rapid polymerase chain reactionbased test for screening Steinert's disease (DM1). Neurol India 2010;58: 99-102.

17. Kakourou G, Dhanjal S, Mamas T, Serhal P, Delhanty JD, SenGupta SB. Modification of the triplet repeat primed polymerase chain reaction method for detection of the CTG repeat expansion in myotonic dystrophy 
type 1: application in preimplantation genetic diagnosis. Fertil Steril 2010;94:1674-9.

18. Day JW, Ricker $K$, Jacobsen JF, Rasmussen LJ, Dick KA, Kress W, et al. Myotonic dystrophy type 2: molecular, diagnostic and clinical spectrum.
Neurology 2003;60:657-64.

19. Mahadevan M, Tsilfidis C, Sabourin L, Shutler G, Amemiya C, Jansen G, et al. Myotonic dystrophy mutation: an unstable CTG repeat in the 3 untranslated region of the gene. Science 1992;255:1253-5. 